\title{
Roots of Two Transcendental Equations Determining the Frequency Spectra of Standing Spherical Electromagnetic Waves*
}

\author{
By Robert L. Pexton and Arno D. Steiger
}

Abstract. Roots of the transcendental equations

$$
\frac{j_{l}(\lambda)}{y_{l}(\lambda)}=\frac{j_{l}(\alpha \lambda) \frac{i_{l-1}(\alpha \lambda \sqrt{|\epsilon|})}{i_{l}(\alpha \lambda \sqrt{|\epsilon|)}}-\frac{1}{\sqrt{|\epsilon|}} j_{l-1}(\alpha \lambda)}{y_{l}(\alpha \lambda) \frac{i_{l-1}(\alpha \lambda \sqrt{|\epsilon|)}}{i_{l}(\alpha \lambda \sqrt{|\epsilon|})}-\frac{1}{\sqrt{|\epsilon|}} y_{l-1}(\alpha \lambda)}
$$

and

$$
\frac{\eta j_{l-1}(\eta)-l j_{l}(\eta)}{\eta y_{l-1}(\eta)-l y_{l}(\eta)}=\frac{\frac{|\epsilon|}{1+|\epsilon|} \alpha \eta j_{l-1}(\alpha \eta)-l j_{l}(\alpha \eta)+\frac{\sqrt{|\epsilon|}}{1+|\epsilon|} \alpha \eta j_{l}(\alpha \eta) \frac{i_{l-1}(\alpha \eta \sqrt{|\epsilon|})}{i_{l}(\alpha \eta \sqrt{|\epsilon|})}}{\frac{|\epsilon|}{1+|\epsilon|} \alpha \eta y_{l-1}(\alpha \eta)-l y_{l}(\alpha \eta)+\frac{\sqrt{|\epsilon|}}{1+|\epsilon|} \alpha \eta y_{l}(\alpha \eta) \frac{i_{l-1}(\alpha \eta \sqrt{|\epsilon|)}}{i_{l}(\alpha \eta \sqrt{|\epsilon|})}}
$$

for the spherical Bessel functions of the first and second kind, $j_{l}(x)$ and $y_{l}(x)$, and for the modified spherical Bessel functions of the first kind, $i_{l}(x)$, have been computed. The ranges for the parameters $\sqrt{|\epsilon|}$ and $\alpha$, the order $l$ and the root index $n$ are:

$$
\sqrt{|\epsilon|}=1.0,10.0,100.0,500.0 ; \quad \alpha=0.1(0.1) 0.7 ; \quad l=1(1) 15 ; \quad n=1(1) 30
$$

In a previous communication [1] roots of two transcendental equations involving spherical Bessel functions were presented. These roots correspond to the eigenfrequency spectra of the transverse electric and the transverse magnetic multipole fields in the domain bounded by two perfectly conducting concentric spheres $(r=$

Received December 14, 1976.

AMS (MOS) subject classifications (1970). Primary 65 A05; Secondary 65H05, 78 A04, $33 \mathrm{A04}$.

Key words and phrases. Roots of transcendental equations, spherical Bessel functions, modified spherical Bessel functions, electromagnetic cavity resonators.

* This work was performed under the auspices of the U.S. Energy Research and Development Administration under contract No. W-7405-Eng-48.

Notice. "This report was prepared as an account of work sponsored by the United States Government. Neither the United States nor the United States Energy Research \& Development Administration, nor any of their employees, nor any of their contractors, subcontractors, or their employees, makes any warranty, express or implied, or assumes any legal liability or responsibility for the accuracy, completeness or usefulness of any information, apparatus, product or process disclosed, or represents that its use would not infringe privately-owned rights." 
$\alpha R$ and $r=R, 0<\alpha<1$ ). On the surface of a perfect conductor the tangential components of the electric field and the normal component of the magnetic field vanish. In the present work these boundary conditions are assumed to hold only on the outer sphere. The inner sphere is regarded as the boundary of a nontransparent plasma core into which the electromagnetic fields penetrate as evanescent (spherical) waves. The necessary and sufficient conditions to ensure continuity of the electromagnetic field are that the tangential components of the electric and magnetic field vectors be continuous across this boundary surface, which is assumed to be charge-free and current-free.

From these boundary conditions then follow the characteristic equations [2]

$$
\frac{j_{l}(\lambda)}{y_{l}(\lambda)}=\frac{\sqrt{\epsilon} j_{l-1}(\alpha \lambda \sqrt{\epsilon}) j_{l}(\alpha \lambda)-j_{l-1}(\alpha \lambda) j_{l}(\alpha \lambda \sqrt{\epsilon})}{\sqrt{\epsilon} j_{l-1}(\alpha \lambda \sqrt{\epsilon}) y_{l}(\alpha \lambda)-y_{l-1}(\alpha \lambda) j_{l}(\alpha \lambda \sqrt{\epsilon})}
$$

and

$$
\begin{aligned}
& \frac{\eta j_{l-1}(\eta)-l j_{l}(\eta)}{\eta y_{l-1}(\eta)-l y_{l}(\eta)} \\
& \quad=\frac{\alpha \eta \sqrt{\epsilon}\left[j_{l-1}(\alpha \eta \sqrt{\epsilon}) j_{l}(\alpha \eta)-\sqrt{\epsilon} j_{l-1}(\alpha \eta) j_{l}(\alpha \eta \sqrt{\epsilon})\right]+(\epsilon-1) l j_{l}(\alpha \eta \sqrt{\epsilon}) j_{l}(\alpha \eta)}{\alpha \eta \sqrt{\epsilon}\left[j_{l-1}(\alpha \eta \sqrt{\epsilon}) y_{l}(\alpha \eta)-\sqrt{\epsilon} y_{l-1}(\alpha \eta) j_{l}(\alpha \eta \sqrt{\epsilon})\right]+(\epsilon-1) l j_{l}(\alpha \eta \sqrt{\epsilon}) y_{l}(\alpha \eta)} .
\end{aligned}
$$

The spherical Bessel functions of the first and second kind, $j_{l}(z)$ and $y_{l}(z)$, are defined in [3, p. 437]. The $n$th root, $\lambda_{l, n}$, of Eq. (1) is proportional to the $n$th characteristic frequency of the transverse electric $2^{l}$-pole field. The $n$th root, $\eta_{l, n}$, of Eq. (2) is proportional to the $n$th characteristic frequency of the transverse magnetic $2^{l}$-pole field. The dielectric constant $\epsilon$ is in general a complex number. If $\epsilon$ is real and negative $(\epsilon=-|\epsilon|)$, the electromagnetic field enters the plasma core as an evanescent wave without being absorbed (total internal reflection). With this choice of $\epsilon$ and the relations [3, pp. 443, 469]

$$
\begin{aligned}
& j_{l}(i z)=e \sqrt[i l \pi / 2]{\frac{\pi}{2 z}} I_{l+1 / 2}(z), \quad(-\pi<\arg z \leqslant 1 / 2 \pi) \\
& i_{l}(x)=\sqrt{\frac{\pi}{2 x}} I_{l+1 / 2}(x)
\end{aligned}
$$

where $I_{l+1 / 2}(x)$ is the modified Bessel function of the first kind, Eqs. (1) and (2) become

$$
\frac{j_{l}(\lambda)}{y_{l}(\lambda)}=\frac{j_{l}(\alpha \lambda) \frac{i_{l-1}(\alpha \lambda \sqrt{|\epsilon|})}{i_{l}(\alpha \lambda \sqrt{|\epsilon|})}-\frac{1}{\sqrt{|\epsilon|}} j_{l-1}(\alpha \lambda)}{y_{l}(\alpha \lambda) \frac{i_{l-1}(\alpha \lambda \sqrt{|\epsilon|})}{i_{l}(\alpha \lambda \sqrt{|\epsilon|})}-\frac{1}{\sqrt{|\epsilon|}} y_{l-1}(\alpha \lambda)}
$$




$$
\frac{\eta j_{l-1}(\eta)-l j_{l}(\eta)}{\eta y_{l-1}(\eta)-l y_{l}(\eta)}
$$

$$
=\frac{\frac{|\epsilon|}{1+|\epsilon|} \alpha \eta j_{l-1}(\alpha \eta)-l j_{l}(\alpha \eta)+\frac{\sqrt{|\epsilon|}}{1+|\epsilon|} \alpha \eta j_{l}(\alpha \eta) \frac{i_{l-1}(\alpha \eta \sqrt{|\epsilon|})}{i_{l}(\alpha \eta \sqrt{|\epsilon|})}}{\frac{|\epsilon|}{1+|\epsilon|} \alpha \eta y_{l-1}(\alpha \eta)-l y_{l}(\alpha \eta)+\frac{\sqrt{|\epsilon|}}{1+|\epsilon|} \alpha \eta y_{l}(\alpha \eta) \frac{i_{l-1}(\alpha \eta \sqrt{|\epsilon|})}{i_{l}(\alpha \eta \sqrt{|\epsilon|})}} .
$$

In the limit $|\epsilon| \rightarrow \infty$ the equations (3) and (4) become, respectively, identical with the equations (1) and $\left(2^{\prime}\right)$ of reference [1].

The calculation of the roots of Eqs. (3) and (4) was performed as follows. First, the equations were each expressed in the form $F(u)=0$. Then, for a given order $l$, a fixed parameter $\alpha$, and a given value for the absolute magnitude of the dielectric constant $\epsilon$, the function $F(u)$ was evaluated at a sequence of points $u_{j}$, for which $u_{j}-u_{j-1}$ $=$ constant, until a sign change occurred. In this last interval a root was computed by using a modified Muller technique [4]. The spherical Bessel functions were computed by means of Mechel's recurrence method [5]. The ratios of the modified spherical Bessel functions were computed using Lentz's continued fraction technique [6] .

The numerical values for the roots of Eqs. (3) and (4) which are listed in the microfiche supplement of this issue are accurate to at least 10 significant figures. The ranges for the square root of the absolute magnitude of the dielectric constant $\epsilon$, the parameter $\alpha$, the order $l$ and the root index $n$ are

$$
\begin{aligned}
\sqrt{|\epsilon|} & =1.0,10.0,100.0,500.0 ; \\
\alpha & =0.1(0.1) 0.7 \\
l & =1(1) 15 \\
n & =1(1) 30
\end{aligned}
$$

These calculations were performed on a CDC 7600 computer.

Lawrence Livermore Laboratory

University of California

Livermore, California 94550

1. R. L. PEXTON \& A. D. STEIGER, "Roots of two transcendental equations involving spherical Bessel functions," Math. Comp., v. 31, 1977, pp.

2. H. L. BOYEN, A. M. MESSIAEN \& P. E. VANDENPLAS, "Experimental and theoretical eigenmodes of a spherical cavity partially filled with plasma," J. Appl. Phys., v. 40, 1969, pp. 2296-2305.

3. M. ABRAMOWITZ \& I. A. STEGUN (Editors), Handbook of Mathematical Functions with Formulas, Graphs, and Mathematical Tables, Nat. Bur. Standards, Appl. Math. Ser., no. 55, Superintendent of Documents, U.S. Government Printing Office, Washington, D.C., 1965. MR 31 \#1400.

4. J. M. BLATT, “A stable method of inverse interpolation," Austral. Comput. J., v. 7, 1975, pp. 51-57. MR 52 \#7077.

5. Fr. MECHEL, "Improvement in recurrence techniques for the computation of Bessel functions of integral order," Math. Comp., v. 22, 1968, pp. 202-205.

6. W. J. LENTZ, "Generating Bessel functions in Mie scattering calculations using continued fractions," Applied Optics, v. 15, 1976, pp. 668-671. 